\section{REVIEW ARTICLE}

\section{I.S. Haldorsen}

A. Espeland

E.-M. Larsson

\title{
Central Nervous System Lymphoma: Characteristic Findings on Traditional and Advanced Imaging
}

SUMMARY: CNS lymphoma consists of 2 major subtypes: secondary CNS involvement by systemic lymphoma and PCNSL. Contrast-enhanced MR imaging is the method of choice for detecting CNS lymphoma. In leptomeningeal CNS lymphoma, representing two-thirds of secondary CNS lymphomas, imaging typically shows leptomeningeal, subependymal, dural, or cranial nerve enhancement. Single or multiple periventricular and/or superficial contrast-enhancing lesions are characteristic of parenchymal CNS lymphoma, representing one-third of secondary CNS lymphomas and almost $100 \%$ of PCNSLs. New CT and MR imaging techniques and metabolic imaging have demonstrated characteristic findings in CNS lymphoma, aiding in its differentiation from other CNS lesions. Advanced imaging techniques may, in the future, substantially improve the diagnostic accuracy of imaging, ultimately facilitating a noninvasive method of diagnosis. Furthermore, these imaging techniques may play a pivotal role in planning targeted therapies, prognostication, and monitoring treatment response.

$\mathbf{L}$ ymphoma of the CNS consists of 2 major subtypes: secondary CNS involvement by systemic lymphoma (the most common) and PCNSL, in which the lymphoma is restricted to the brain, leptomeninges, spinal cord, or eyes, without evidence of it outside the CNS at primary diagnosis. ${ }^{1,2}$

The frequency of secondary CNS lymphoma in patients with systemic lymphoma varies and is highly dependent on histologic subtype. The overall risk of CNS relapse in aggressive NHL is on the order of $2 \%-27 \%,{ }^{3,4}$ whereas patients with Hodgkin lymphoma are at very low risk $(\leq 0.5 \%) .{ }^{3}$ Patients with extranodal involvement and those with primary or acquired immunodeficiency disorders carry an increased risk of CNS relapse. ${ }^{3}$

PCNSL accounts for $1 \%-5 \%$ of all brain tumors ${ }^{5,6}$ and approximately $1 \%$ of all NHLs. ${ }^{5}$ The incidence rates of PCNSL are increasing among immunocompetent patients. ${ }^{5,7}$ Immunocompromised patients (eg, individuals infected with HIV) have an increased risk of developing PCNSL. ${ }^{9}$ However, with the introduction of HAART during the past decade, the incidence of PCNSL in the HIV population has declined. ${ }^{10,11}$

Early diagnosis of CNS lymphoma is crucial for proper management in both immunocompetent and immunocom-

From the Department of Radiology (I.S.H., A.E.), Haukeland University Hospital, Bergen, Norway; Section for Radiology (I.S.H., A.E.), Department of Surgical Sciences, University of Bergen, Bergen, Norway; and Department of Radiology (E.-M.L.), Uppsala University Hospital, Uppsala, Sweden

Please address correspondence to Ingfrid S. Haldorsen, MD, PhD, Department of Radiology, Haukeland University Hospital, Jonas Liesvei 65, 5021 Bergen, Norway; e-mail: ingfrid. haldorsen@helse-bergen.no

Indicates open access to non-subscribers at www.ajnr.org

DOI 10.3174/ajnr.A2171 promised individuals and is more likely if a tumor is observed on imaging. ${ }^{12,13}$ Although CNS lymphomas may have characteristic imaging findings on traditional CT and MR imaging, none of these will unequivocally differentiate CNS lymphoma from other brain lesions. ${ }^{9,14-16}$ A visible tumor on imaging is essential to raise the suspicion of CNS lymphoma, which then can lead to an early histologic diagnosis based on cytology of the CSF or brain biopsy.

This pictorial essay reviews some typical imaging features at the presentation of CNS lymphoma on traditional imaging. Characteristic imaging findings with newer advanced imaging techniques that may potentially aid in the differentiation of CNS lymphoma from other brain lesions are also discussed.

\section{Secondary CNS Lymphoma: Traditional Imaging}

CNS involvement in aggressive NHL tends to occur early at a median of 5-12 months after the primary diagnosis of NHL. ${ }^{3}$ Approximately two-thirds of the patients present with leptomeningeal spread and one-third, with parenchymal disease. ${ }^{3}$ Leptomeningeal spread, similar to leptomeningeal metastases from any cause, often involves the cranial nerves, spinal cord, or spinal roots and may present as cranial or spinal neuropathies. ${ }^{17}$ Headache is present in $30 \%-40 \%$ of patients with leptomeningeal metastases, ${ }^{18}$ which may be due to increased intracranial pressure from metastatic obstruction of CSF flow/absorption. ${ }^{17}$

The imaging technique of choice to detect leptomeningeal metastasis is contrast-enhanced MR imaging. ${ }^{17,18} \mathrm{CT}$ is less sensitive, ${ }^{18}$ especially in patients with hematologic malignancies. ${ }^{19}$ Neuroimaging findings suggestive of leptomeningeal metastases include leptomeningeal, subependymal, dural, or cranial nerve enhancement; superficial cerebral lesions; and 
Table 1: Typical imaging features of primary and secondary CNS lymphoma

\begin{tabular}{|c|c|c|}
\hline & Primary CNS lymphoma & Secondary CNS Lymphoma \\
\hline \multirow[t]{2}{*}{ Primary site of CNS involvement } & Brain parenchyma, $\sim 100 \%$ & Brain parenchyma, $\sim 1 / 3$; leptomeninges, $\sim 2 / 3$ \\
\hline & Parenchymal CNS Iymphoma & Leptomeningeal CNS Iymphoma \\
\hline Typical location & Periventricular and superficial brain regions & Leptomeninges \\
\hline CT findings & Iso- or hypodense lesions with marked CE & $\begin{array}{l}\text { Leptomeningeal, subependymal, dural, or cranial nerve } \\
\text { CE; superficial cerebral lesion; communicating } \\
\text { hydrocephalus }\end{array}$ \\
\hline MRI findings & $\begin{array}{l}\text { T1: hypo- or isointense lesions, moderate-marked CE } \\
\text { T2: iso- or hyperintense lesions; often hypointense to } \\
\text { gray matter }\end{array}$ & $\begin{array}{l}\text { Leptomeningeal, subependymal, dural, or cranial nerve } \\
\text { CE; superficial cerebral lesion; communicating } \\
\text { hydrocephalus }\end{array}$ \\
\hline Enhancement pattern & Non-AIDS patients: homogeneous CE, 90\%; & Leptomeningeal, subependymal, dural, or cranial nerve CE \\
\hline
\end{tabular}

Enhancement pattern

ring-CE, $\sim 0 \%-13 \%$

AIDS patients: irregular CE common; ring-CE, $\sim 75 \%$
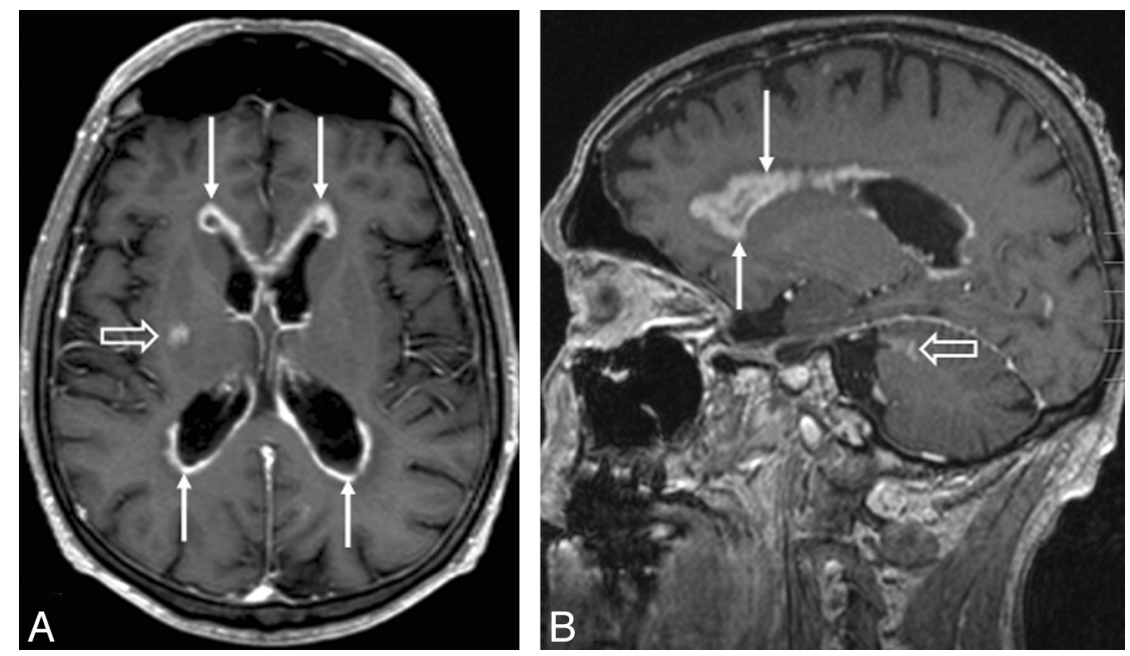

Fig 1. Axial $(A)$ and sagittal $(B)$ T1-weighed contrast-enhanced MR images in a patient with CNS metastases from NHL show diffuse subependymal contrast enhancement (arrows) and 2 parenchymal lesions (open arrows) in the right basal ganglia $(A)$ and left cerebellum $(B)$.

communicating hydrocephalus (Table 1). ${ }^{17,18,20,21}$ Lumbar puncture may induce dural enhancement in the cranial or spinal space, which may be falsely interpreted as leptomeningeal enhancement. Lumbar puncture should, therefore, be avoided before neuroimaging. ${ }^{18}$

Parenchymal metastases from NHL often appear as single or multiple enhancing lesions and can be accompanied by leptomeningeal metastases. ${ }^{17,21}$ The parenchymal lesions may have a periventricular and/or superficial location (Fig 1). The typical MR imaging findings are quite similar to the findings in PCNSL; this similarity makes it impossible to discriminate these 2 entities on the basis of neuroimaging. ${ }^{16}$ As for all primary brain tumors and brain metastases, MR imaging is the imaging technique of choice for the detection of lesions and preoperative planning of surgical diagnostic procedures.

Approximately $50 \%$ of the patients with CNS metastases from NHL have progressive systemic lymphoma at the time of diagnosis of their CNS manifestation. ${ }^{3}$ Furthermore, most of the remaining $50 \%$ of patients with apparently isolated CNS metastases develop systemic disease within months. ${ }^{3}$ Systemic manifestations of NHL thus commonly accompany the findings of parenchymal or leptomeningeal disease; these manifestations normally raise clinical suspicion of CNS metastases and lead to early cytology or brain biopsy to confirm the diagnosis.

\section{PCNSL: Conventional CT and MR Imaging}

PCNSL often has a characteristic appearance on both CT and MR imaging (Table 1). This is due to its hypercellularity, high nuclear/cytoplasmic ratio, disruption of the blood-brain barrier, and its predilection for the periventricular and superficial regions, often in contact with ventricular or meningeal surfaces. $22-25$

In both immunocompetent and immunodeficient patients with PCNSL, unenhanced CT typically reveals hyper- or isoattenuated lesions, and virtually all lesions show contrast enhancement. ${ }^{24-27}$ Negative CT findings, however, do not exclude PCNSL because false-negative initial CT examinations are also reported. ${ }^{14,25}$ On unenhanced T1-weighted MR imaging, lesions are typically hypo- or isointense, ${ }^{14,24-27}$ and on T2-weighted MR imaging, iso- to hyperintense but often hypointense to gray matter. ${ }^{14,25,26,28}$ Most lesions show moderate-to-marked contrast enhancement on CT and MR imaging. ${ }^{14,15,24-27,29}$ Isolated white matter hyperintensity on T2-weighted MR imaging or no contrast enhancement on T1weighted MR imaging has also been described in some rare cases of PCNSL. ${ }^{14,30}$ 

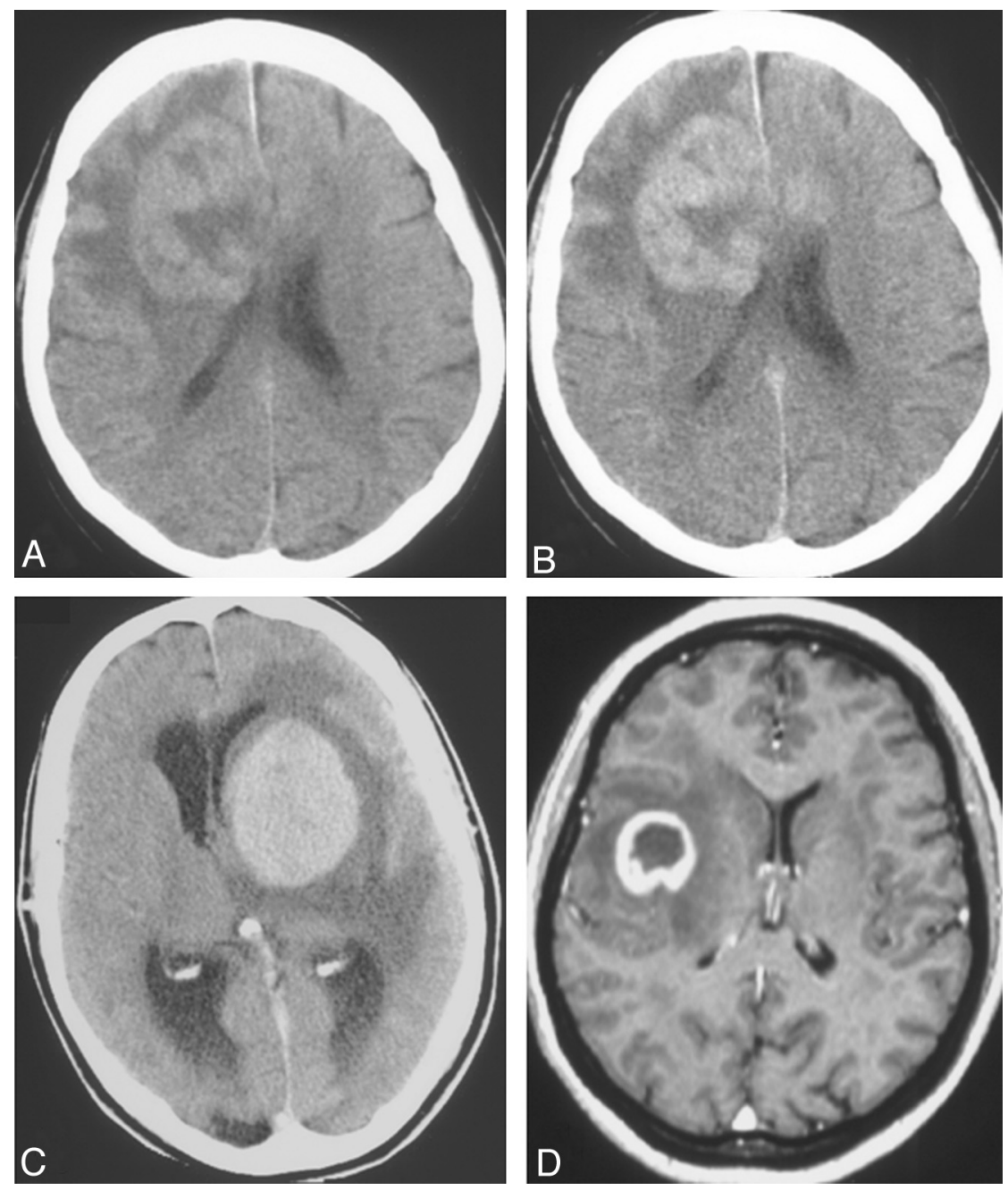

Fig 2. Solitary lesions on a noncontrast CT scan $(A)$, contrast-enhanced CT scans ( $B$ and $C$ ), and an MR image $(D)$ in 3 patients with non-AIDS PCNSL. Note a hyperattenuated lesion in the frontal lobe on the noncontrast CT scan $(A)$ with marked enhancement on the contrast series $(B)$ and focal contrast-enhancing lesions in the left basal ganglia $(C)$ and temporal lobe $(D)$. One lesion has ring enhancement $(D)$.

Non-AIDS PCNSL typically presents as a solitary homogeneously enhancing parenchymal mass (Fig 2). ${ }^{14}$ Multiple lesions are reported in $20 \%-40 \%$ of non-AIDS PCNSLs, ${ }^{14,15,27,29,31}$ and ringlike enhancement, in $0 \%-13 \%$ (Figs 2 and 3). ${ }^{14,29,31,32}$ Linear enhancement along perivascular spaces is highly suggestive of PCNSL. ${ }^{23,25}$ Perifocal edema is usually present but less prominent than that in malignant gliomas or metastases. ${ }^{14,33}$ Most lesions are located in central hemispheric or periventricular cerebral white matter. ${ }^{14,25,34} \mathrm{~A}$ superficial location adjacent to the meninges is also common. Frontal lobe location is reported in $20 \%-43 \%$ of PCNSLs, whereas the basal ganglia are affected in $13 \%-20 \% .{ }^{14,26,32,35}$ The brain stem or cerebellum or both are affected in 9\%$13 \%,{ }^{15,32,35}$ and the spinal cord, in only $1 \%-2 \%$ of patients. ${ }^{15,29}$ Hemorrhage or internal calcification within the tumor is quite a rare finding. ${ }^{14,27}$

An extension of parenchymal PCNSL to the eye, denoting secondary intraocular lymphoma, may be asymptomatic and has been reported in $25 \%$ of the PCNSLs. ${ }^{36}$ It may be diagnosed by cytologic examination of vitreal aspirate or by slit-lamp examination. ${ }^{13,23}$ Primary intraocular lym- phoma, the lymphoma being solely restricted to the eye, is a very rare subset of PCNSL. ${ }^{13}$ Using a dedicated thinsection MR imaging protocol, intraocular lymphoma may be detected as nodular contrast-enhancing lesions at the macula or thickening of the uvea. ${ }^{37}$ However, MR imaging has a low sensitivity for intraocular lymphoma, and negative findings do not preclude the intraocular involvement of PCNSL. ${ }^{37}$

Primary dural lymphoma is a rare subtype of PCNSL that differs biologically from other PCNSLs because it arises from the dura mater. ${ }^{38} \mathrm{CT}$ or MR imaging typically reveals diffusely enhancing single or multiple extra-axial masses, which often mimic meningiomas (Fig 4). ${ }^{38}$ The cerebral convexities are most commonly involved; however, dural lymphoma may also be found in the falx, tentorium, sellar/parasellar regions, or spine. ${ }^{38}$

Immunodeficient patients with PCNSL are often diagnosed with multifocal lesions, which are reported in 30\%$80 \%$ of patients with AIDS-related PCNSL (Fig 5). ${ }^{28,31,39}$ Because many lesions exhibit necrotic regions, contrast enhancement is commonly irregular or peripheral, and ringlike 

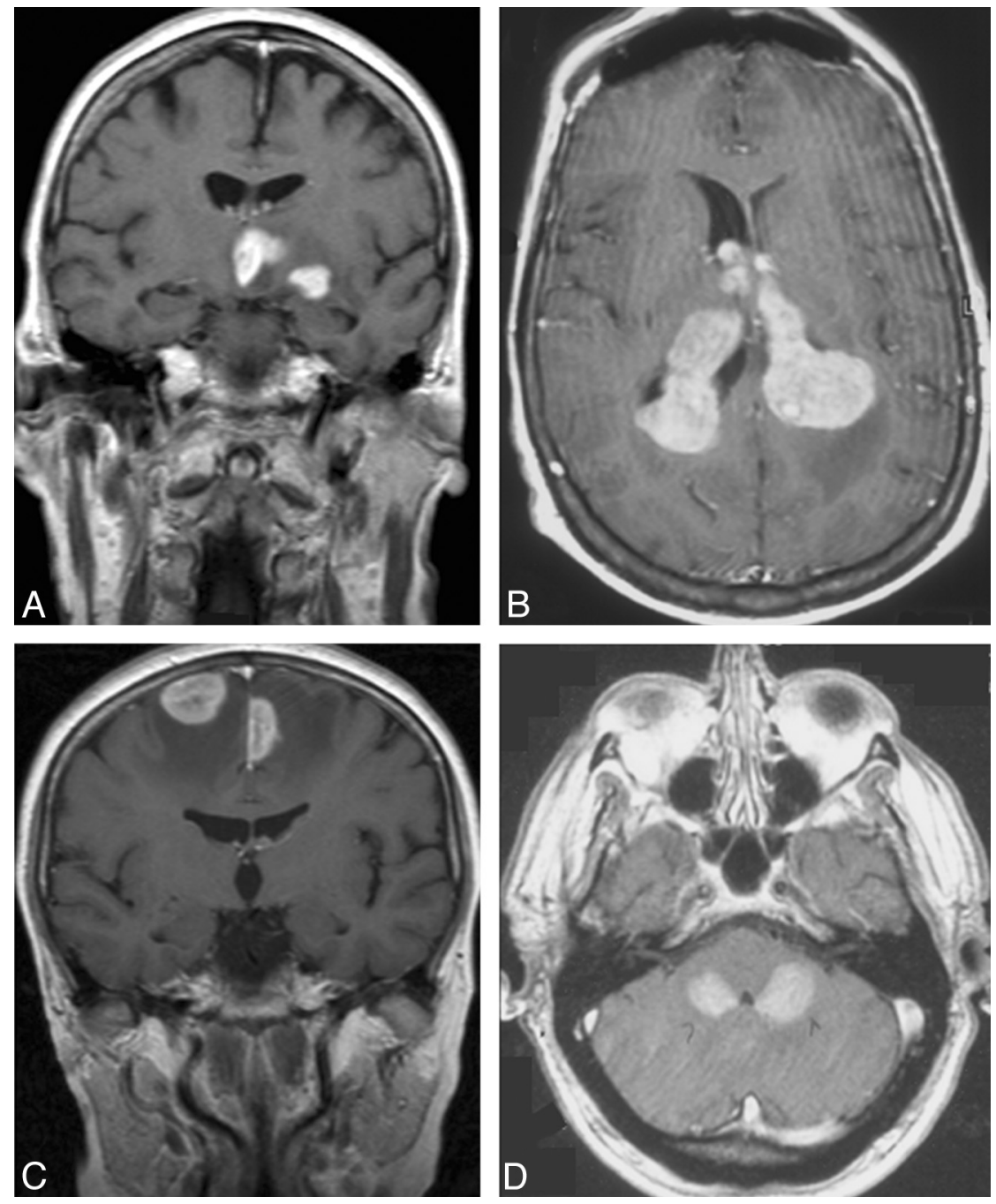

Fig 3. Multiple lesions on contrast-enhanced axial $(B$ and $D)$ and coronal $(A$ and $C)$ MR images in 4 patients with non-AIDS PCNSL. Note lesions in the basal ganglia $(A)$, ventricles $(B)$, frontal lobes $(C)$, and cerebellar lobes $(D)$.

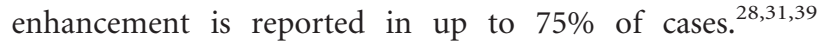
The basal ganglia and corpus callosum are frequently involved. ${ }^{28,34,39}$ Spontaneous hemorrhage in PCNSL lesions may be more frequent in AIDS patients than in non-AIDS patients. $^{28}$

\section{CNS Lymphoma: Advanced CT and MR Imaging}

CNS lymphomas may have a characteristic appearance on traditional CT and MR imaging; however, none of these imaging characteristics will unequivocally differentiate CNS lymphomas from other neoplasms (eg, metastases from other malignancies, malignant gliomas, meningiomas) or non-neoplastic diseases (eg, multiple sclerosis, stroke, cerebral toxoplasmosis, pyogenic abscess). ${ }^{9,14-16}$ Furthermore, the typical imaging characteristics may not be present. ${ }^{14} \mathrm{DWI},{ }^{25,40}$ perfusion MR imaging, ${ }^{40,41}$ and MR spectroscopy ${ }^{42,43}$ are increasingly used in clinical radiologic practice and may help to differentiate CNS lymphomas from other lesions of the brain (Table 2).

DWI measures the diffusion of water molecules in biologic tissues; diffusion within the tumor is considered a surrogate marker of tumor cellularity because intact cells constitute a barrier to water diffusion. ${ }^{44}$ Because CNS lymphomas are highly cellular tumors, water diffusion is often restricted, making them appear hyperintense on DWI and hypointense on ADC maps (Figs 4 and 6). ${ }^{16,40,44,45}$ This characteristic is shared by acute ischemic stroke, the central necrosis of brain abscesses, the solid portion of high-grade gliomas, and some metastases. ${ }^{45}$ However, PCNSL lesions often have more restricted diffusion and lower ADC values than high-grade gliomas and metastases. ${ }^{40,46,47}$

A recent study showed that pretherapeutic ADC tumor measurements within contrast-enhancing regions were predictive of clinical outcome in patients with PCNSL. ${ }^{48}$ Low ADC values were predictive of shorter progression-free survival and overall survival. In addition, an inverse correlation was found between ADC values and the cellular density of the tumors. Patients with prolonged progression-free survival and overall survival also had a significant reduction in post-therapeutic ADC values. ${ }^{48}$ Thus, repeated ADC measurements may be used as biomarkers in the surveillance of therapeutic response.

Diffusion tensor imaging requires diffusion measurements in at least 6 directions and is a sensitive tool for the detection of alterations in white matter structure. ${ }^{47}$ A quantitative FA map shows hypointensity corresponding to decreased FA values in 

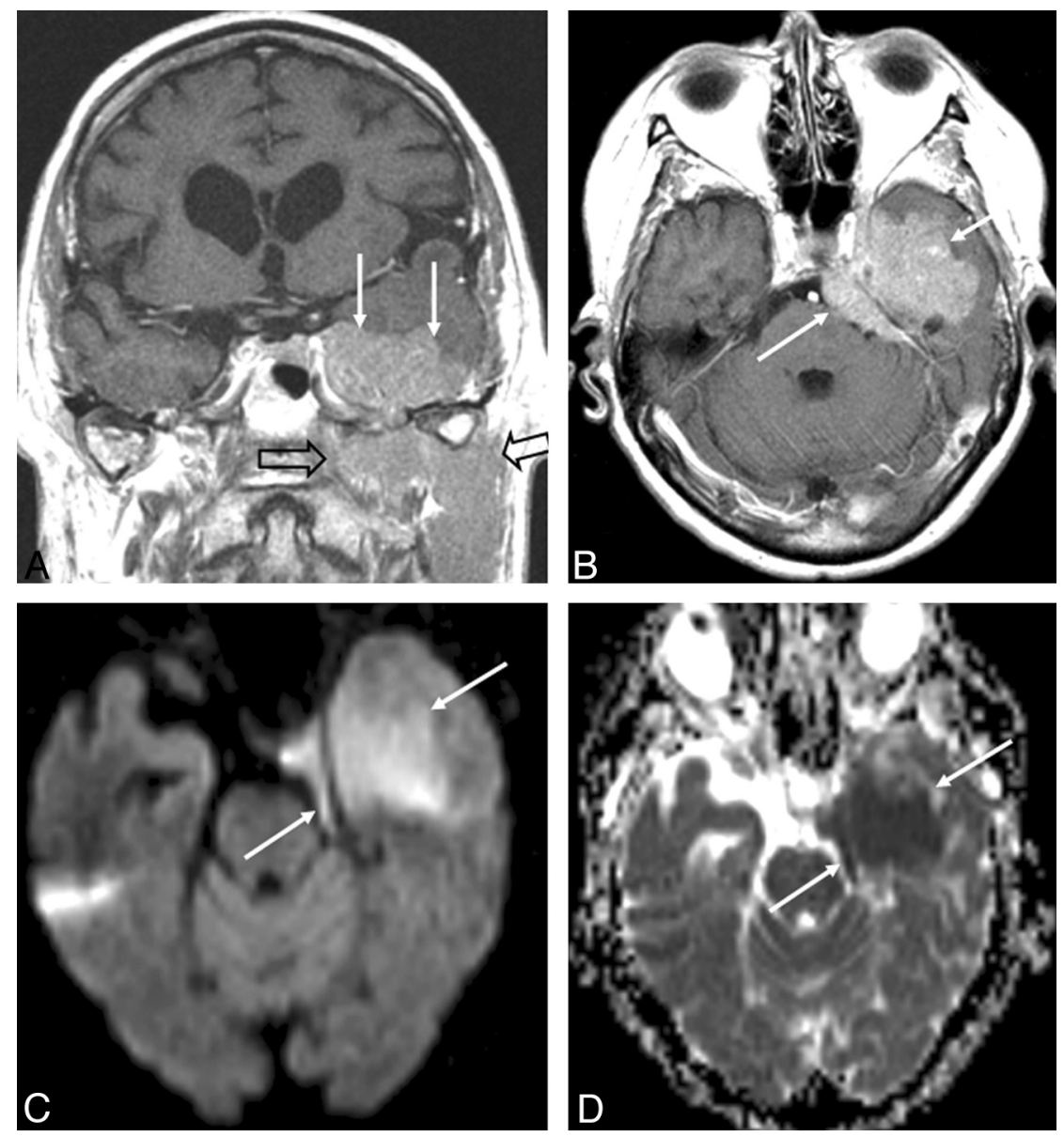

Fig 4. Coronal $(A)$ and axial $(B)$ contrast-enhanced T1-weighted MR images and an axial DWI $(C)$ and $A D C$ map $(D)$ in a patient with primary dural B-cell lymphoma (arrows) with tumor spread below the skull base (open arrow). The contrast-enhancing tumor at the caudal surface of the left temporal lobe $(A$ and $B)$ displaces the temporal lobe cranially and resembles a meningioma. DWI reveals restricted diffusion within the tumor with high signal intensity on $D W I(C)$ and corresponding low signal intensity on the $A D C$ map $(D)$.

most brain tumors. ${ }^{47}$ Different degrees of cellularity and cellular organization may also affect the FA value, and FA values of PCNSL are significantly lower than those of glioblastoma multiforme, aiding in the differentiation of these tumors. ${ }^{47}$

The documented importance of revascularization through angiogenesis for tumor growth has led to a growing interest in novel imaging techniques to assess tumor vascularity. Perfusion MR imaging and perfusion CT visualize nutritive delivery of arterial blood to the capillary bed in the biologic tissue (eg, tumors); postprocessing of the acquired data enables calculation of physiologic parameters, such as $\mathrm{CBV}$, cerebral blood flow, mean transit time, and time to peak. ${ }^{49-51}$ PCNSLs demonstrate low CBV (Fig 6) and a characteristic intensity time curve, which is related to a massive leakage of contrast media into the interstitial space. ${ }^{41}$ Furthermore, maximum relative $\mathrm{CBV}$ measured in tumor tissue, calculated as a ratio to contralateral normal-appearing white matter, is typically lower in lymphomas than in other brain tumors. This characteristic finding can help to differentiate glioblastomas and metastases from lymphomas. ${ }^{40,52}$

Both perfusion MR imaging and perfusion CT may demonstrate increased microvascular permeability in tumor tissue based on quantification of the permeability surface area product and the contrast transfer coefficient. ${ }^{50,53}$ Several studies have reported that these parameters measured on MR imaging correlate with the mitotic index, histologic grading, and biologic aggressiveness of gliomas, but this correlation has not yet been investigated with regard to CNS lymphomas. ${ }^{50}$ In contrast to MR imaging, only a few studies on perfusion CT in brain tumors ${ }^{50,51}$ have been published, possibly due to concern about the radiation dose with CT and potential nephrotoxicity of iodinated contrast agents. ${ }^{50}$ However, the diagnostic value of perfusion CT in brain tumors may become more important in the future because it offers advantages over perfusion MR imaging in terms of spatial resolution, insensitivity to paramagnetic susceptibility artifacts, and linear correlation between contrast concentration and attenuation. ${ }^{50}$

MR spectroscopy obtains biochemical information noninvasively from biologic tissue. Within a defined volume of interest, signals may be registered from chemical nuclei within the body; the most commonly used nuclei are protons (hydrogen). In PCNSL, proton MR spectroscopy has demonstrated elevated lipid peaks combined with high Cho/Cr ratios. ${ }^{29,42-44}$ These can, however, also be seen in glioblastoma multiforme ${ }^{42}$ and metastases but may help in differentiating PCNSL from other lesions.

High-resolution SWI is much more sensitive than conventional MR imaging for the visualization of small veins, blood products, and calcifications, which appear as low-signal intensity structures. ${ }^{54}$ This is helpful in differentiating PCNSL 

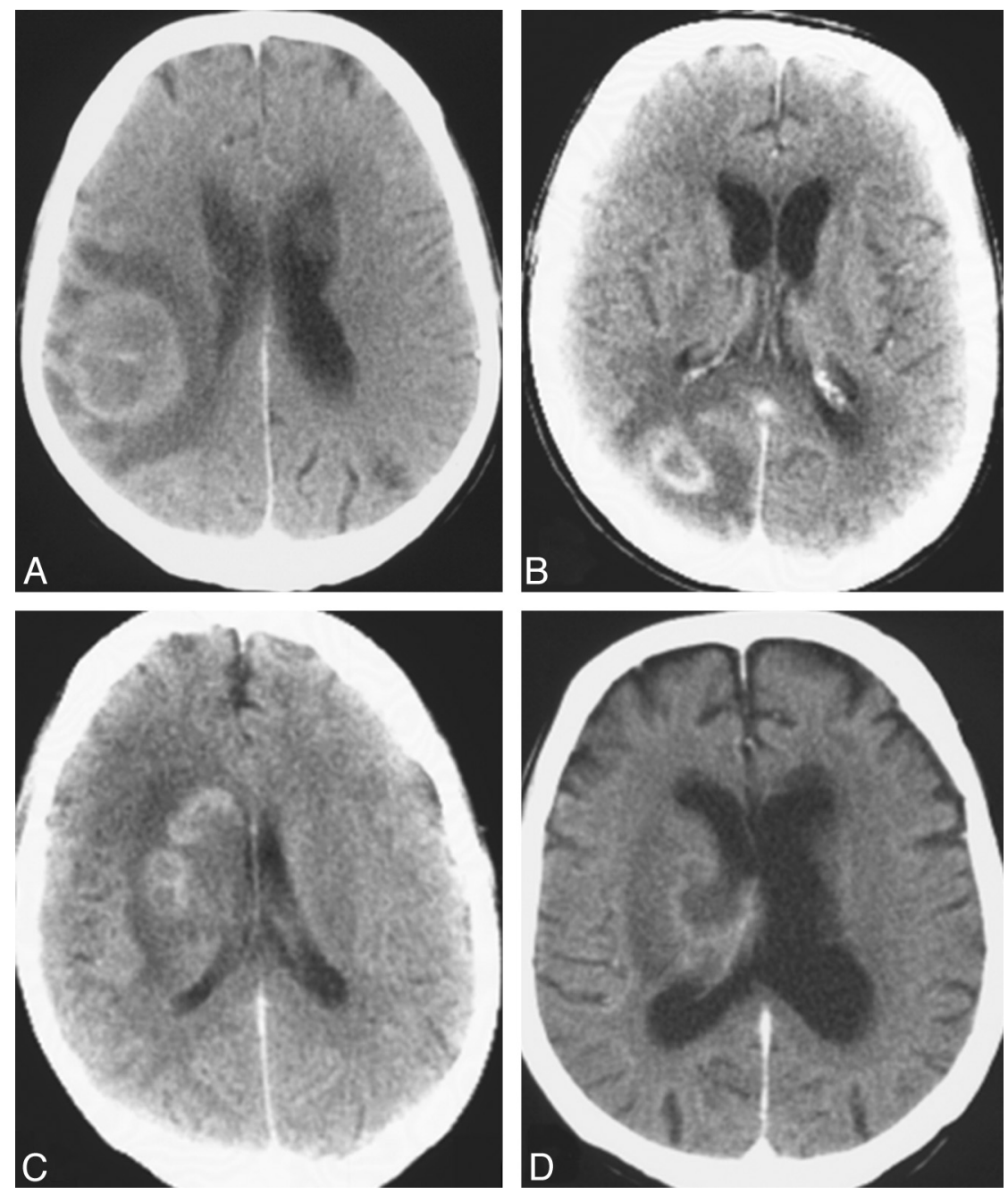

Fig 5. Contrast-enhancing lesions on CT scans $(A-D)$ in 4 patients with AIDS-related PCNSL. Note irregularly enhancing lesions in the right parietal lobe $(A)$, right occipital lobe $(B)$, and right periventricular white matter $(C$ and $D)$; most of the lesions show ring enhancement $(A, B$, and $C)$.

\begin{tabular}{|c|c|c|}
\hline Imaging Method & Findings in CNS Lymphoma & Potential Value as Diagnostic Tool in CNS Lymphoma \\
\hline \multicolumn{3}{|l|}{$\begin{array}{l}\mathrm{MRI} / \mathrm{CT} \\
\end{array}$} \\
\hline $\begin{array}{l}\text { Diffusion MRI/diffusion tensor } \\
\text { imaging }\end{array}$ & $\begin{array}{l}\text { Restricted diffusion in lesions (hyperintense on DWI and } \\
\text { hypointense on ADC maps) })^{40,44-47} \\
\text { Decreased FA values in lesions }{ }^{47}\end{array}$ & $\begin{array}{l}\text { Differentiation of CNS Iymphoma and malignant glioma/ } \\
\text { metastases }\end{array}$ \\
\hline $\begin{array}{l}\text { Perfusion MRI/perfusion CT/ } \\
\text { permeability MRI }\end{array}$ & $\begin{array}{l}\text { Low maximum } \mathrm{CBV}^{40,41} \\
\text { Characteristic intensity time curve related to leakage of } \\
\text { contrast into the interstitial space }{ }^{41}\end{array}$ & $\begin{array}{l}\text { Differentiation of CNS Iymphoma and malignant glioma/ } \\
\text { metastases } \\
\text { Assessment of microvascular tumor permeability relevant } \\
\text { for diagnosis, prognostication, and therapy }\end{array}$ \\
\hline MR spectroscopy & Elevated lipid peaks and high $\mathrm{Cho} / \mathrm{Cr}$ ratios ${ }^{29,42-44,59}$ & $\begin{array}{l}\text { Differentiation of CNS lymphoma and some gliomas and } \\
\text { CNS lymphomas and toxoplasmosis/PML in AIDS } \\
\text { patients }\end{array}$ \\
\hline High-resolution SWI & Blood products and calcifications are rare findings ${ }^{14,27}$ & Differentiation of CNS lymphoma and high-grade gliomas \\
\hline MRI with new contrast agents & $\begin{array}{l}\text { MRI with iron oxide nanoparticles: lesions enhance less } \\
\text { than with gadolinium }{ }^{56}\end{array}$ & Differentiation of CNS lymphoma and MS \\
\hline \multicolumn{3}{|l|}{ Metabolic imaging } \\
\hline PET & $\begin{array}{l}\text { FDG-PET, methionine PET: hypermetabolic lesions with } \\
\text { increased uptake of FDG or methionine } e^{25,60,62}\end{array}$ & $\begin{array}{l}\text { Differentiation of CNS lymphoma and gliomas/metastases/ } \\
\text { meningiomas } \\
\text { Early evaluation of therapeutic response }\end{array}$ \\
\hline SPECT/SPET & $\begin{array}{l}\text { Hypermetabolic lesions with high } N \text {-isopropyl } \\
\text { iodoamphetamine or thallium-201 uptake }{ }^{9,63,64}\end{array}$ & $\begin{array}{l}\text { Differentiation of AIDS-related CNS lymphoma and } \\
\text { infectious intracranial lesions }\end{array}$ \\
\hline
\end{tabular}

from high-grade gliomas, because microhemorrhages and calcifications are rare in PCNSL, whereas small hemorrhages are frequently seen in high-grade gliomas. ${ }^{54}$
More recently, superparamagnetic contrast agents, (eg, iron oxide nanoparticles) have become available. ${ }^{55} \mathrm{MR}$ imaging with iron oxide nanoparticles may help to distinguish ma- 

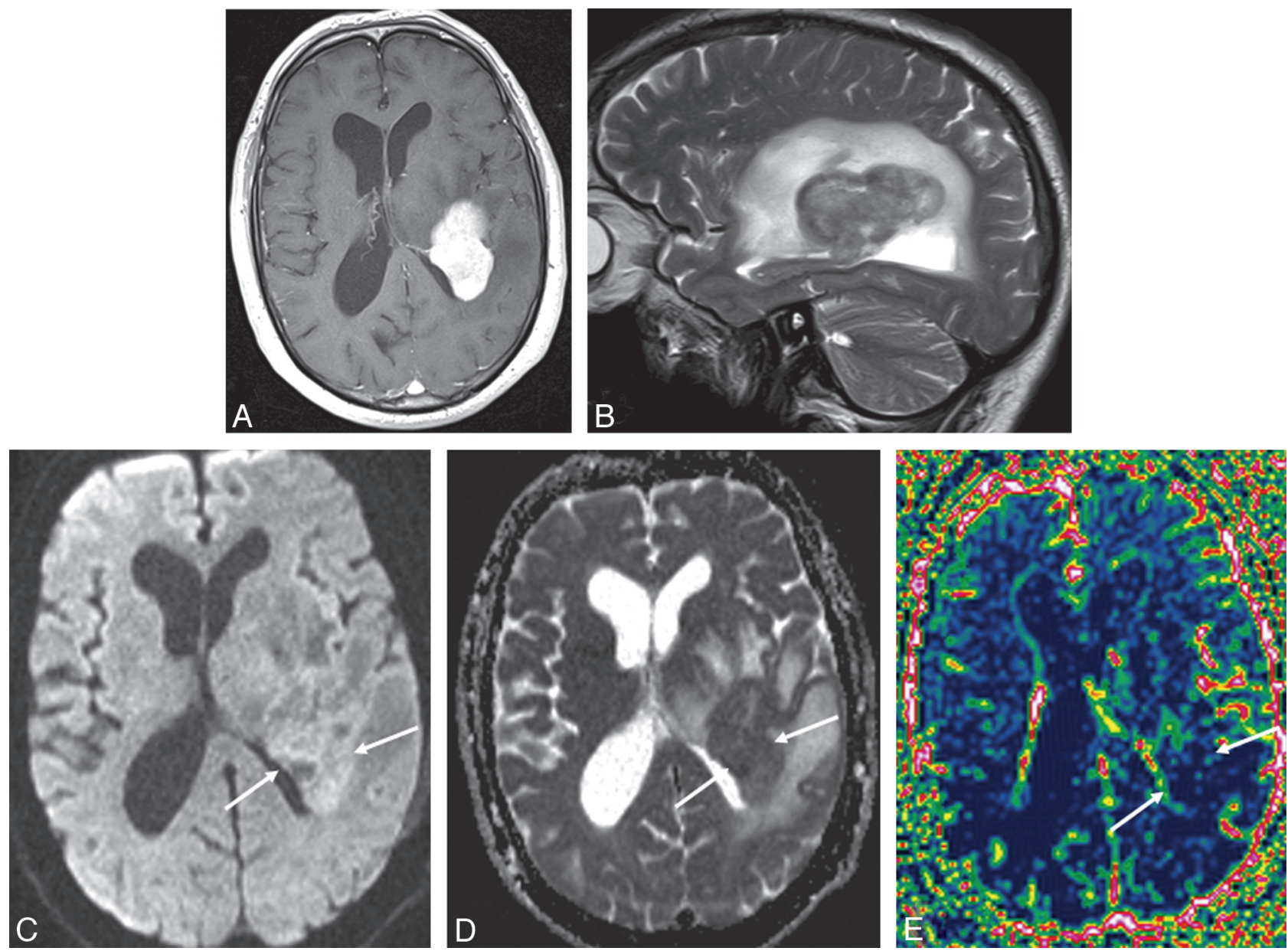

Fig 6. Axial contrast-enhanced T1-weighted MR image $(A)$, sagittal T2-weighted MR image $(B)$, axial DWI $(C)$, an $A D C$ map $(D)$, and an rCBV map $(E)$ in a patient with PCNSL. The periventricular contrast-enhancing tumor $(A)$ in the left parietal lobe has restricted diffusion with high signal intensity on DWI $(C)$ with corresponding low signal intensity on the $A D C$ map (arrows, D). Perfusion MR imaging shows low perfusion within the contrast-enhancing tumor on the rCBV map (arrows, E).

lignant neoplasms from multiple sclerosis ${ }^{56}$; however, to our knowledge, the potential role of this technique in the diagnosis of CNS lymphoma has not yet been defined.

In AIDS patients, DWI cannot reliably differentiate CNS lymphomas from cerebral toxoplasmosis because they show overlapping ADC ratios. ${ }^{45}$ Perfusion MR imaging has also been disappointing in this respect. ${ }^{57}$ However, on MR spectroscopy, toxoplasmosis, lymphoma, and PML brain lesions display distinctly different biochemical profiles, ${ }^{58,59}$ aiding in correctly diagnosing $94 \%$ of the lesions in 1 study. ${ }^{59}$

\section{CNS Lymphoma: Metabolic Imaging}

Medical nuclear imaging techniques by using radioisotopes to produce images that reflect biologic processes are examples of metabolic imaging. PET with FDG typically reveals hypermetabolic lesions with an increased uptake of FDG in CNS lymphomas ${ }^{25}$ and may help to identify and differentiate lymphomas from malignant gliomas and meningiomas (Table 2). ${ }^{60}$ PCNSLs show more pronounced metabolic activity than do metastases and high-grade gliomas. ${ }^{25}$ Furthermore, FDG-PET may be suitable for early evaluation of a therapeutic response. ${ }^{60}$ After steroid treatment, the degree of hypermetabolic activity in PCNSL may decrease. ${ }^{61}$

PET performed with ${ }^{11} \mathrm{C}$-methionine (methionine $\mathrm{PET}$ ) shows very high uptakes in CNS lymphomas, which corre- sponds to the enhancing portion on CT/MR imaging, and the area of increased uptake is often larger than the enhancing lesions (Fig 7). ${ }^{62}$ This larger area of methionine uptake reflects tumor infiltration beyond the enhancing portion seen on MR imaging and CT. ${ }^{62}$ The size and degree of methionine accumulation in the tumor tissue decrease following radiation therapy. ${ }^{62}$ Thus, methionine PET may provide a more accurate delineation of tumor volume for the evaluation of the therapeutic effect of radiation therapy as well as for the detection of residual or recurrent tumor after treatment. ${ }^{62}$ SPECT in non-AIDS PCNSL has shown high iodine 123 $\mathrm{N}$-isopropyl-p-iodoamphetamine retention in CNS lesions, aiding in the diagnosis of PCNSL. ${ }^{63}$

Among immunocompromised individuals, infectious lesions in the CNS are usually hypometabolic, whereas lesions caused by CNS lymphomas are hypermetabolic, with a high thallium-201 uptake ratio on SPECT and SPET ${ }^{9,64}$ and high FDG uptake on PET. ${ }^{9}$ These characteristics may aid in the differentiation of infectious intracranial lesions and CNS lymphomas in AIDS patients. ${ }^{9,57,64}$

\section{Future Perspectives}

New PET, SPECT, and SPET tracers as well as new MR imaging contrast agents that may potentially reveal important aspects of tumor biology are currently being intensively in- 

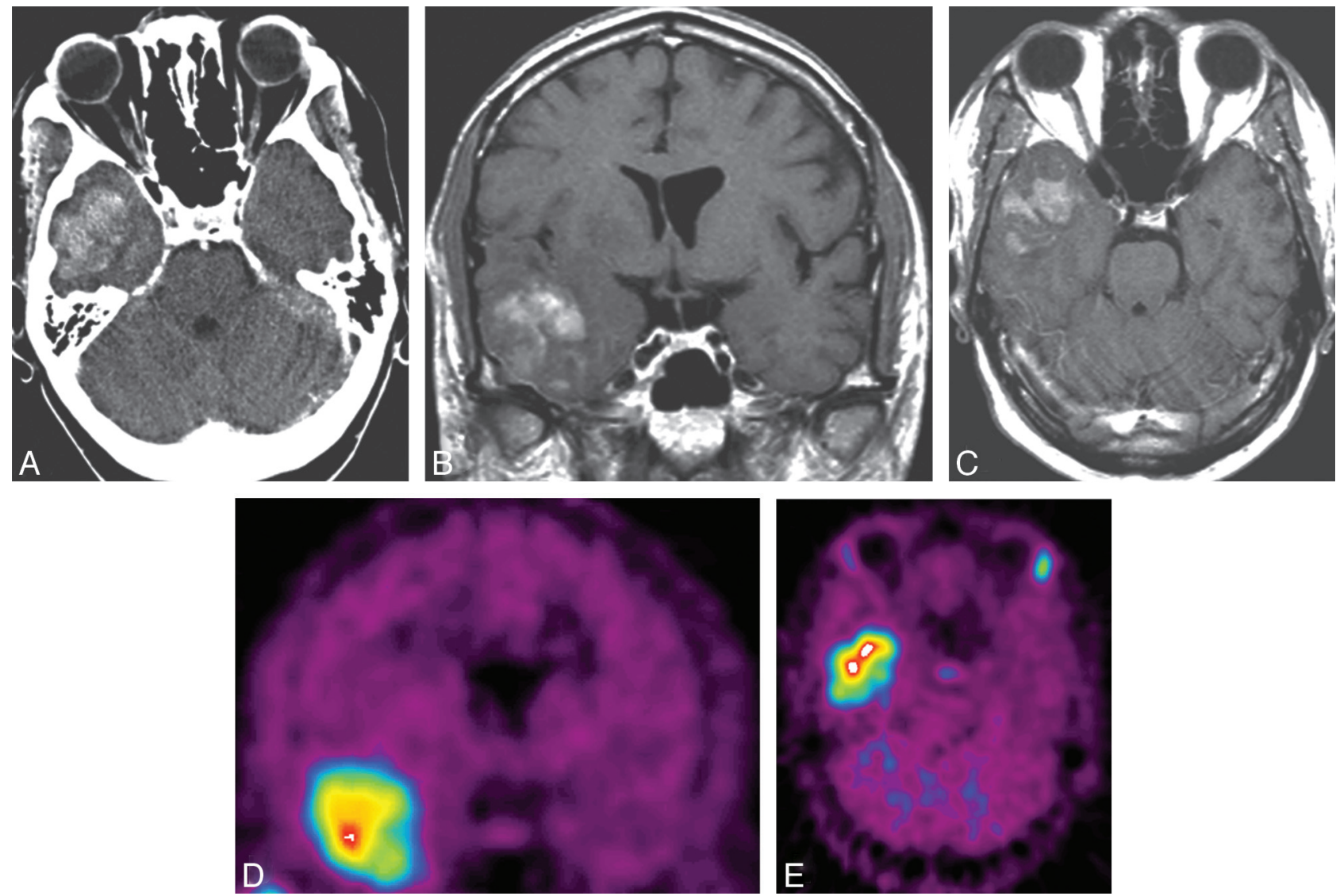

Fig 7. Axial contrast-enhanced CT scan $(A)$, coronal $(B)$ and axial $(C)$ contrast-enhanced T1-weighted MR images, and methionine PET (coronal, $D$, and axial, $E)$ images in a patient with PCNSL. The contrast-enhancing tumor in the right temporal lobe $(A-C)$ shows high uptake of methionine on PET $(D$ and $E)$.

vestigated by researchers all over the world. ${ }^{55,65}$ This research may provide new insights that will substantially improve the preoperative diagnostic accuracy of imaging, enabling an appropriate diagnostic procedure as well as early active treatment in this patient group. In the future, improved advanced imaging techniques may noninvasively provide an accurate diagnosis, obviating surgical biopsy before the initiation of chemotherapy, radiation therapy, and new nonsurgical therapeutic regimens. An integrated PET/MR imaging system has recently been developed that gives simultaneous morphologic and biologic information, creating new possibilities for complementary information. ${ }^{66}$ Some of these newer imaging techniques will presumably play a pivotal role in the planning of new targeted therapies, in monitoring treatment response, and in the prediction of treatment outcomes.

\section{Conclusions}

When CNS lymphoma is suspected, contrast-enhanced MR is the imaging technique of choice. Secondary CNS lymphomas present as leptomeningeal metastases in two-thirds of the patients and as parenchymal metastases in one-third. In PCNSL, almost all patients have parenchymal lesions. Parenchymal lymphomas have a predilection for the periventricular and superficial regions, often abutting the ventricular or meningeal surfaces. Although CNS lymphomas may have characteristic imaging findings on traditional MR imaging, none of these will unequivocally differentiate CNS lymphoma from other brain lesions.
New advanced MR imaging techniques and PET and SPECT metabolic imaging have identified characteristic findings in CNS lymphoma that may aid in the differentiation of CNS lymphomas and other CNS lesions. In the future, improved advanced imaging techniques may provide morphologic and biologic information noninvasively and, thus, an accurate diagnosis. Furthermore, these imaging techniques will presumably play an important role in the planning of new targeted therapies, for prognostication, and for the monitoring of treatment response.

\section{References}

1. Wong ET. Management of central nervous system lymphomas using monoclonal antibodies: challenges and opportunities. Clin Cancer Res 2005;11: $7151 \mathrm{~s}-57 \mathrm{~s}$

2. Mohile NA, Abrey LE. Primary central nervous system lymphoma. Semin Radiat Oncol 2007;17:223-29

3. Hill QA, Owen RG. CNS prophylaxis in lymphoma: who to target and what therapy to use. Blood Rev 2006;20:319-32

4. Montoto S, Lister TA. Secondary central nervous system lymphoma: risk factors and prophylaxis. Hematol Oncol Clin North Am 2005;19:751-63

5. Haldorsen IS, Krossnes BK, Aarseth JH, et al. Increasing incidence and continued dismal outcome of primary central nervous system lymphoma in Norway 1989-2003: time trends in a 15-year national survey. Cancer 2007;110:1803-14

6. van der Sanden GA, Schouten LJ, van Dijck JA, et al. Primary central nervous system lymphomas: incidence and survival in the Southern and Eastern Netherlands. Cancer 2002;94:1548-56

7. Olson JE, Janney CA, Rao RD, et al. The continuing increase in the incidence of primary central nervous system non-Hodgkin lymphoma: a surveillance, epidemiology, and end results analysis. Cancer 2002;95:1504-10

8. Cote TR, Manns A, Hardy CR, et al. Epidemiology of brain lymphoma among people with or without acquired immunodeficiency syndrome: AIDS/Cancer Study Group. J Natl Cancer Inst 1996;88:675-79 
9. Kasamon YL, Ambinder RF. AIDS-related primary central nervous system lymphoma. Hematol Oncol Clin North Am 2005;19:665-87

10. Diamond C, Taylor TH, Aboumrad T, et al. Changes in acquired immunodeficiency syndrome-related non-Hodgkin lymphoma in the era of highly active antiretroviral therapy: incidence, presentation, treatment, and survival. Cancer 2006;106:128-35

11. Besson C, Goubar A, Gabarre J, et al. Changes in AIDS-related lymphoma since the era of highly active antiretroviral therapy. Blood 2001;98:2339-44

12. Haldorsen IS, Espeland A, Larsen JL, et al. Diagnostic delay in primary central nervous system lymphoma. Acta Oncol 2005;44:728-34

13. Morris PG, Abrey LE. Therapeutic challenges in primary CNS lymphoma. Lancet Neurol 2009;8:581-92

14. Haldorsen IS, Krakenes J, Krossnes BK, et al. CT and MR imaging features of primary central nervous system lymphoma in Norway, 1989-2003. AJNR Am J Neuroradiol 2009;30:744-51

15. Buhring $U$, Herrlinger $U$, Krings $T$, et al. MRI features of primary central nervous system lymphomas at presentation. Neurology 2001;57:393-96

16. Senocak E, Oguz KK, Ozgen B, et al. Parenchymal lymphoma of the brain on initial MR imaging: a comparative study between primary and secondary brain lymphoma. Eur J Radiol 2010 Mar 2. [Epub ahead of print]

17. Bierman P, Giglio P. Diagnosis and treatment of central nervous system involvement in non-Hodgkin's lymphoma. Hematol Oncol Clin North Am 2005; 19:597-609

18. DeAngelis LM, Boutros D. Leptomeningeal metastasis. Cancer Invest 2005;23: $145-54$

19. van Oostenbrugge RJ, Twijnstra A. Presenting features and value of diagnostic procedures in leptomeningeal metastases. Neurology 1999;53:382-85

20. Freilich RJ, Krol G, DeAngelis LM. Neuroimaging and cerebrospinal fluid cytology in the diagnosis of leptomeningeal metastasis. Ann Neurol 1995;38: $51-57$

21. Gleissner B, Chamberlain M. Treatment of CNS dissemination in systemic lymphoma. J Neurooncol 2007;84:107-17

22. Fitzsimmons A, Upchurch $\mathrm{K}$, Batchelor T. Clinical features and diagnosis of primary central nervous system lymphoma. Hematol Oncol Clin North Am 2005; 19:689-703

23. Eichler AF, Batchelor TT. Primary central nervous system lymphoma: presentation, diagnosis and staging. Neurosurg Focus 2006;21:E15

24. Koeller KK, Smirniotopoulos JG, Jones RV. Primary central nervous system lymphoma: radiologic-pathologic correlation. Radiographics 1997;17:1497-526

25. Go JL, Lee SC, Kim PE. Imaging of primary central nervous system lymphoma. Neurosurg Focus 2006;21:E4

26. Gliemroth J, Kehler U, Gaebel C, et al. Neuroradiological findings in primary cerebral lymphomas of non-AIDS patients. Clin Neurol Neurosurg 2003;105: $78-86$

27. Coulon A, Lafitte F, Hoang-Xuan K, et al. Radiographic findings in $\mathbf{3 7}$ cases of primary CNS lymphoma in immunocompetent patients. Eur Radiol 2002;12:329-40

28. Thurnher MM, Rieger A, Kleibl-Popov C, et al. Primary central nervous system lymphoma in AIDS: a wider spectrum of CT and MRI findings. Neuroradiology 2001;43:29-35

29. Kuker W, Nagele T, Korfel A, et al. Primary central nervous system lymphomas (PCNSL): MRI features at presentation in 100 patients. J Neurooncol 2005;72:169-77

30. Weaver JD, Vinters HV, Koretz B, et al. Lymphomatosis cerebri presenting as rapidly progressive dementia. Neurologist 2007;13:150-53

31. Fine HA, Mayer RJ. Primary central nervous system lymphoma. Ann Intern Med 1993;119:1093-104

32. Hayakawa T, Takakura K, Abe H, et al. Primary central nervous system lymphoma in Japan: a retrospective, co-operative study by CNS-Lymphoma Study Group in Japan. J Neurooncol 1994;19:197-215

33. Schlegel U, Schmidt-Wolf IG, Deckert M. Primary CNS lymphoma: clinical presentation, pathological classification, molecular pathogenesis and treatment. J Neurol Sci 2000;181:1-12

34. Erdag N, Bhorade RM, Alberico RA, et al. Primary lymphoma of the central nervous system: typical and atypical CT and MR imaging appearances. AJR Am J Roentgenol 2001;176:1319-26

35. Bataille B, Delwail V, Menet E, et al. Primary intracerebral malignant lymphoma: report of 248 cases. J Neurosurg 2000;92:261-66

36. Gunduz K, Pulido JS, McCannel CA, et al. Ocular manifestations and treatment of central nervous system lymphomas. Neurosurg Focus 2006;21:E9

37. Kuker W, Herrlinger U, Gronewaller E, et al. Ocular manifestation of primary nervous system lymphoma: what can be expected from imaging? J Neurol 2002;249:1713-16

38. Iwamoto FM, Abrey LE. Primary dural lymphomas: a review. Neurosurg Focus 2006;21:E5

39. Haldorsen IS, Krakenes J, Goplen AK, et al. AIDS-related primary central ner- vous system lymphoma: a Norwegian national survey 1989-2003. BMC Cancer 2008;8:225

40. Calli $\mathrm{C}$, Kitis $\mathrm{O}$, Yunten N, et al. Perfusion and diffusion MR imaging in enhancing malignant cerebral tumors. Eur J Radiol 2006;58:394-403

41. Hartmann M, Heiland S, Harting I, et al. Distinguishing of primary cerebral lymphoma from high-grade glioma with perfusion-weighted magnetic resonance imaging. Neurosci Lett 2003;338:119-22

42. Harting I, Hartmann M, Jost G, et al. Differentiating primary central nervous system lymphoma from glioma in humans using localised proton magnetic resonance spectroscopy. Neurosci Lett 2003;342:163-66

43. Taillibert S, Guillevin R, Menuel C, et al. Brain lymphoma: usefulness of the magnetic resonance spectroscopy. J Neurooncol 2008;86:225-29

44. Zacharia TT, Law M, Naidich TP, et al. Central nervous system lymphoma characterization by diffusion-weighted imaging and MR spectroscopy. $\mathrm{JNeu}$ roimaging 2008;18:411-17

45. Schroeder PC, Post MJ, Oschatz E, et al. Analysis of the utility of diffusionweighted MRI and apparent diffusion coefficient values in distinguishing central nervous system toxoplasmosis from lymphoma. Neuroradiology 2006; 48:715-20

46. Stadnik TW, Chaskis C, Michotte A, et al. Diffusion-weighted MR imaging of intracerebral masses: comparison with conventional MR imaging and histologic findings. AJNR Am J Neuroradiol 2001;22:969-76

47. Toh $\mathrm{CH}$, Castillo M, Wong AM, et al. Primary cerebral lymphoma and glioblastoma multiforme: differences in diffusion characteristics evaluated with diffusion tensor imaging. AJNR Am J Neuroradiol 2008;29:471-75

48. Barajas RF Jr, Rubenstein JL, Chang JS, et al. Diffusion-weighted MR imaging derived apparent diffusion coefficient is predictive of clinical outcome in primary central nervous system lymphoma. AJNR Am J Neuroradiol 2010;31: 60-66. Epub 2009 Sep 3

49. Wu O, Ostergaard L, Sorensen AG. Technical aspects of perfusion-weighted imaging. Neuroimaging Clin N Am 2005;15:623-37, xi

50. Cianfoni A, Colosimo C, Basile M, et al. Brain perfusion CT: principles, technique and clinical applications. Radiol Med 2007;112:1225-43

51. Fainardi E, Di BF, Borrelli M, et al. Potential role of CT perfusion parameters in the identification of solitary intra-axial brain tumor grading. Acta Neurochir Suppl 2010;106:283-87

52. Hakyemez B, Erdogan C, Bolca N, et al. Evaluation of different cerebral mass lesions by perfusion-weighted MR imaging. J Magn Reson Imaging 2006; 24:817-24

53. Larsson HB, Courivaud F, Rostrup E, et al. Measurement of brain perfusion, blood volume, and blood-brain barrier permeability, using dynamic contrast-enhanced T(1)-weighted MRI at 3 Tesla. Magn Reson Med 2009;62: $1270-81$

54. Kim HS, Jahng GH, Ryu CW, et al. Added value and diagnostic performance of intratumoral susceptibility signals in the differential diagnosis of solitary enhancing brain lesions: preliminary study. AJNR Am J Neuroradiol 2009;30: 1574-79

55. Peng XH, Qian X, Mao H, et al. Targeted magnetic iron oxide nanoparticles for tumor imaging and therapy. Int J Nanomedicine 2008;3:311-21

56. Manninger SP, Muldoon LL, Nesbit G, et al. An exploratory study of ferumoxtran-10 nanoparticles as a blood-brain barrier imaging agent targeting phagocytic cells in CNS inflammatory lesions. AJNR Am J Neuroradiol 2005;26:2290-300

57. Bakshi R. Neuroimaging of HIV and AIDS related illnesses: a review. Front Biosci 2004;9:632-46

58. Chang L, Ernst T. MR spectroscopy and diffusion-weighted MR imaging in focal brain lesions in AIDS. Neuroimaging Clin N Am 1997;7:409-26

59. Chang L, Miller BL, McBride D, et al. Brain lesions in patients with AIDS: H-1 MR spectroscopy. Radiology 1995;197:525-31

60. Palmedo $\mathrm{H}$, Urbach $\mathrm{H}$, Bender $\mathrm{H}$, et al. FDG-PET in immunocompetent patients with primary central nervous system lymphoma: correlation with MRI and clinical follow-up. Eur J Nucl Med Mol Imaging 2006;33:164-68

61. Rosenfeld SS, Hoffman JM, Coleman RE, et al. Studies of primary central nervous system lymphoma with fluorine-18-fluorodeoxyglucose positron emission tomography. J Nucl Med 1992;33:532-36

62. Ogawa T, Kanno I, Hatazawa J, et al. Methionine PET for follow-up of radiation therapy of primary lymphoma of the brain. Radiographics $1994 ; 14$ 101-10

63. Shinoda J, Yano H, Murase S, et al. High 123I-IMP retention on SPECT image in primary central nervous system lymphoma. J Neurooncol 2003;61:261-65

64. Ruiz A, Ganz WI, Post MJ, et al. Use of thallium-201 brain SPECT to differentiate cerebral lymphoma from toxoplasma encephalitis in AIDS patients. AJNR Am J Neuroradiol 1994;15:1885-94

65. Chen W, Silverman DH. Advances in evaluation of primary brain tumors. Semin Nucl Med 2008;38:240-50

66. Heiss WD. The potential of PET/MR for brain imaging. Eur J Nucl Med Mol Imaging 2009;36(suppl 1):S105-12 